\title{
温控近红外光谱用于葡萄糖的高灵敏检测
}

\author{
汪明圆崔晓宇蔡文生郡学广* \\ (南开大学化学学院 天津市生物传感与分子识别重点实验室 天津 300071)
}

\begin{abstract}
摘要 温控近红外光谱在混合体系定量分析以及分子间相互作用分析中已得到应用. 本工作利用互因子分析方法 (MFA)探索了温控近红外光谱技术测定血清样品中低浓度葡萄糖含量的可行性. 测量了不同温度下葡萄糖含量分别为 $1.0 \sim 15.0 \mathrm{mmol} / \mathrm{L}$ 和 $0.0 \sim 1.0 \mathrm{mmol} / \mathrm{L}$ 的两组血清溶液的近红外光谱, 并利用 $\mathrm{MFA}$ 进行了定量计算. 结果表明, 利用该 方法可以实现样品中微量葡萄糖的准确定量, MFA 提取的标准信号 $(\mathbf{S S})$ 的相对强度和葡萄糖浓度之间的线性相关系数 $R$ 分别为 0.9923 和 0.9895 , 预测均方根误差(RMSEP)分别为 0.35 和 $0.07 \mathrm{mmol} / \mathrm{L}$.
\end{abstract}

关键词 近红外光谱; 温度效应; 互因子分析; 葡萄糖检测; 血清

\section{Temperature-Dependent Near-Infrared Spectroscopy for Sensitive Detection of Glucose}

\author{
Wang, Mingyuan Cui, Xiaoyu Cai, Wensheng Shao, Xueguang* \\ (College of Chemistry, Nankai University, Tianjin Key Laboratory of Biosensing and Molecular Recognition, \\ Tianjin 300071, China)
}

\begin{abstract}
Temperature-dependent near-infrared (NIR) spectroscopy has been proposed and used in the quantitative analysis of multi-component mixtures and the understanding of the interactions in solutions. Mutual factor analysis (MFA) was developed, in our previous work, to detect glucose content in aqueous solutions and serum samples using the NIR spectra measured at different temperatures. The essence of the algorithm is to extract and compare the spectral component, named as standardized signal (SS), mutually contained in the spectral data of different samples. The relative quantity of SS can be used to build the calibration model for quantitative analysis. Furthermore, the spectral information of water can be used for the analysis, because the change of the water spectrum with temperature is a reflection of the change in glucose content. In this work, serum samples with low glucose concentration were prepared and measured at the temperature range of $30 \sim 60{ }^{\circ} \mathrm{C}$ with a step of $5{ }^{\circ} \mathrm{C}$. The feasibility of MFA in the quantitative determination of low concentration samples was further studied. Serum solutions with glucose content of $1.0 \sim 15.0 \mathrm{mmol} / \mathrm{L}$ and $0.0 \sim 1.0 \mathrm{mmol} / \mathrm{L}$ were prepared, respectively. Before calculation of MFA, continuous wavelet transform (CWT) was used to improve the resolution of the spectra. The results show that MFA can achieve an accurate quantification of the glucose content. The linear correlation coefficients $(R)$ of the calibration models between the relative quantity of SS and the concentration of glucose are 0.9923 and 0.9895 , respectively, and the root-mean-squared error of prediction (RMSEP) are 0.35 and $0.07 \mathrm{mmol} / \mathrm{L}$, respectively. The relative error of predicted concentration of samples in the validation set obtained from the calibration model of samples with a concentration of $1.0 \sim 15.0$ $\mathrm{mmol} / \mathrm{L}$ are in the range of $-12.00 \% \sim 5.64 \%$, which are in a reasonable level for clinical uses. Temperature-dependent NIR spectroscopy combined with MFA may be a potential way for detecting the micro-content components in complex aqueous systems.
\end{abstract}

Keywords near-infrared spectroscopy; temperature effect; mutual factor analysis; glucose detection; serum

\section{1 引言}

近红外光谱技术在研究以水为基质的生物或环境 样品方面发挥着重要作用, 在葡萄糖含量检测中的应用 已有报道 ${ }^{[1,2]}$, 如利用近红外光谱技术测定水溶液 ${ }^{[3 \sim 7]}$ 、 含有蛋白质的葡萄糖水溶液 ${ }^{[8]}$ 、血清 ${ }^{[6,7,9 \sim 11]}$ 以及全血 ${ }^{[12]}$ 中的葡萄糖含量. Arnold 课题组利用偏最小二乘算法 $(\mathrm{PLS})$ 分别研究了葡萄糖水溶液中 $1.25 \sim 19.66 \mathrm{mmol} / \mathrm{L}$
葡萄糖 ${ }^{[3]}$ 、含有牛血清白蛋白的葡萄糖水溶液中 $1.2 \sim$ $20.0 \mathrm{mmol} / \mathrm{L}$ 葡萄糖 ${ }^{[8]}$ 、未稀释人血清中 $3.16 \sim 31.86$ $\mathrm{mmol} / \mathrm{L}$ 葡萄糖 ${ }^{[11]}$ 以及牛全血中 $3 \sim 30 \mathrm{mmol} / \mathrm{L}$ 葡萄糖 ${ }^{[12]}$ 的定量, 其中血清和全血中葡萄糖定量模型的预测标准 偏差(SEP)分别为 1.29 和 $0.96 \mathrm{mmol} / \mathrm{L}$. Ozaki 课题组 ${ }^{[13]}$ 采用搜索组合移动窗口偏最小二乘算法(SCMWPLSR) 对通过向牛血清中添加葡萄糖得到牛血清溶液中 1.66 17.67 mmol/L 葡萄糖进行了定量研究, 获得的预

*E-mail: xshao@nankai.edu.cn

Received December 12, 2019; published February 13, 2020.

Project supported by the National Natural Science Foundation of China (No. 21775076) and the Fundamental Research Funds for the Central Universities (No. 63191743).

项目受国家自然科学基金(No. 21775076)和中央高校基本科研基金项目(No. 63191743)资助. 
测模型的预测均方根误差 $(\mathrm{RMSEP})$ 为 $0.57 \mathrm{mmol} / \mathrm{L}$. 这 些研究主要利用了近红外光谱一级倍频和组合频区域 中葡萄糖 $\mathrm{CH}$ 或 $\mathrm{OH}$ 特征吸收峰的信息, 避免了体系中 水的特征吸收区域. 本课题组利用水对温度扰动、溶质 浓度变化敏感的特点 ${ }^{[14,15]}$, 以水为探针研究了葡萄糖水 溶液以及人血清中葡萄糖的定量 ${ }^{[6,7]}$, 模型的相关系数 分别为 0.99 和 0.93 , 为水基质样品的定量分析提供了一 种新方法.

近红外光谱的温度效应一直是建立定量模型的难 题. 研究发现在使用 $8300 \sim 5500 \mathrm{~cm}^{-1}$ 波段对葡萄糖浓 度进行定量分析时, $1{ }^{\circ} \mathrm{C}$ 的温度变化就会导致葡萄糖浓 度预测误差大约为 $27.78 \mathrm{mmol} / \mathrm{L}^{[16]}$. 为了校正温度效 应, Hazen 等 ${ }^{[3]}$ 提出了结合偏最小二乘的傅里叶滤波方 法, 并利用对温度不敏感光谱区域的信息进行葡萄糖定 量. Tarumi 等 ${ }^{[16]}$ 将散射效应、温度效应等因素纳入校准 模型中, 以降低预测误差. 本课题组利用近红外光谱的 温度效应在定性和定量分析方面开展了研究工作, 分析 了水溶液中葡萄糖 ${ }^{[17]}$ 、寡肽 ${ }^{[18]}$ 、聚合物 ${ }^{[19]}$ 和水的相互作 用, 胺类化合物的氢键相互作用 ${ }^{[20]}$, 研究了溶液和血清 中蛋白质热变性过程中蛋白质和水化层的结构变 化 ${ }^{[21,22]}$, 并实现了以水结构的变化监测卵清蛋白凝胶化 过程 ${ }^{[23]}$. 此外, 通过温控近红外光谱还实现了烷烃体 系、乙醇水溶液、葡萄糖水溶液以及血清样品的定量分 析 $^{[6,7,24 \sim 26]}$.

互因子分析(Mutual Factor Analysis, 简称 MFA)方 法 ${ }^{[7]}$ 的本质是提取和比较不同样品光谱数据中共同包含 的光谱信息, 利用该算法已实现了葡萄糖含量分别为 $111.01 \sim 444.05 \mathrm{mmol} / \mathrm{L}$ 和 $5.00 \sim 16.65 \mathrm{mmol} / \mathrm{L}$ 的水溶液 中葡萄糖的准确定量, 并且以葡萄糖含量为 $4.55 \sim 8.05$ $\mathrm{mmol} / \mathrm{L}$ 血清样品的光谱对定量模型进行了外部验证, 得到的 RMSEP 值为 $0.46 \mathrm{mmol} / \mathrm{L}$, 证明了 MFA 方法用 于葡萄糖检测的可行性和适用性. 本文中, 进一步研究 了利用 MFA 算法实现温控近红外光谱对血清样品中低 浓度范围葡萄糖检测的可行性, 测定了以血清为溶剂不 同浓度范围的葡萄糖溶液 $(1.0 \sim 15.0 \mathrm{mmol} / \mathrm{L}$ 和 $0.0 \sim 1.0$ $\mathrm{mmol} / \mathrm{L}$ )在不同温度下的近红外光谱. 采用 $\mathrm{MFA}$ 方法提 取与葡萄糖浓度变化相关的光谱, 对样品中葡萄糖含量 进行准确定量.

\section{2 结果与讨论}

\section{1 光谱分析}

分别测量了以血清为溶剂, 葡萄糖浓度为 $1.0 \sim$ $15.0 \mathrm{mmol} / \mathrm{L}$ (A 组样品)和 $0.0 \sim 1.0 \mathrm{mmol} / \mathrm{L}$ (B 组样品) 在不同温度下的近红外光谱, 测量温度范围为 $30 \sim$ $60{ }^{\circ} \mathrm{C}$, 升温间隔为 $5{ }^{\circ} \mathrm{C}$. 图 1 (a)为葡萄糖浓度分别为 0 和 $15 \mathrm{mmol} / \mathrm{L}$ 样品在 $30 、 45$ 和 $60{ }^{\circ} \mathrm{C}$ 下的近红外光谱. 研 究表明溶质会对溶剂结构产生影响 ${ }^{[17,18]}$, 因此本文分析 了水在 $8000 \sim 6000 \mathrm{~cm}^{-1}$ 范围内的光谱, 利用不同温度
下水的光谱信息实现样品中葡萄糖的定量检测. 可以发 现, $6900 \mathrm{~cm}^{-1}$ 附近存在一个宽峰, 其光谱特征对应于含 有不同氢键的水结构中 $\mathrm{OH}$ 伸缩振动的倍频吸收 ${ }^{[17,23]}$. 通过对比两个样品在不同温度下的光谱可以明显看出 随着温度升高, 吸收峰峰位置明显向高波数移动, 表明 水分子的结构及不同结构形式水分子的含量发生了变 化, 尤其是氢键发生了变化 ${ }^{[6]}$. 然而, 由于由葡萄糖浓 度引起的光谱差异很小并且光谱成分重叠严重, 很难通 过识别特征峰来实现葡萄糖含量的定量.
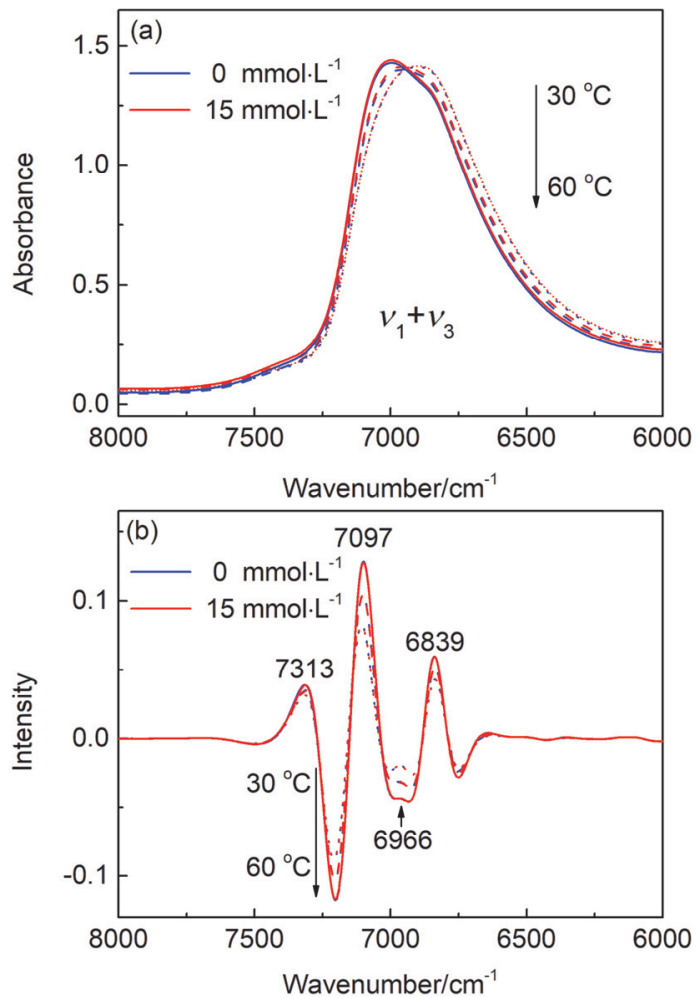

图 1 葡萄糖浓度分别为 0 (蓝色) 和 $15 \mathrm{mmol} / \mathrm{L}$ (红色)样品在 30 (点线)、 45 (虚线)和 $60{ }^{\circ} \mathrm{C}$ (实线)下的(a)原始光谱和(b)小波变换光谱图(Sym6) Figure 1 Measured spectra (a) and transformed spectra (b) of serum with the glucose content of 0 (blue) and $15.0 \mathrm{mmol} / \mathrm{L}$ (red), measured at 30 (dot line), 45 (dash line) and 60 (solid line) ${ }^{\circ} \mathrm{C}$, respectively. Transformed spectra were calculated by CWT with Sym6.

为了提高光谱的分辨率，使用 “Sym6” 为滤波器的 连续小波变换 (Continuous Wavelet Transform, 简称 CWT)计算了原始光谱的近似六阶导数 ${ }^{[27]}$. 图 1(b)显示 了与图 1(a)中光谱对应的小波变换结果. 可以发现, 在 $7313,7097,6966$ 和 $6839 \mathrm{~cm}^{-1}$ 附近存在 4 个峰, 前 2 个 吸收峰可以分别归属为水中 $\mathrm{OH}$ 转动吸收峰以及自由 $\mathrm{OH}$ 对称伸缩和反对称伸缩振动的组合频吸收峰，后两 个吸收峰为含有不同氢键的水结构中 $\mathrm{OH}$ 伸缩振动的倍 频吸收峰 ${ }^{[14,15,17,27]}$. 显然, 随着温度的升高含有不同氢

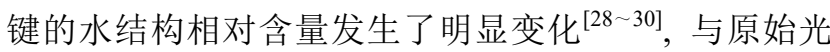
谱相比较, 温度效应在小波变换后的光谱中体现得更为 显著，这种高分辨信息有可能为葡萄糖的定量分析提供 
更多帮助.

\section{2 定量分析}

为了进行定量计算, 将不同样品在不同温度下测得 的光谱按照浓度横向排列构成组合数据矩阵, 采用 MFA 得到标准信号(SS). 根据 MFA 的原理, SS 体现了 光谱随温度的变化, 而 SS 在不同浓度样品光谱中的相 对强度(即 $z_{i}$ 值) 体现了浓度的相对高低. 通过葡萄糖浓 度与 $z_{i}$ 之间建立的定量模型即可实现样品中葡萄糖的定 量分析. 图 2 展示了浓度为 $1.0 \sim 15.0 \mathrm{mmol} / \mathrm{L}$ 的样品光 谱数据的 MFA 计算结果. 图 2(a)为标准信号 SS 的谱图, 嵌入图为 SS 在 $7097 \mathrm{~cm}^{-1}$ 处的峰强度与温度之间的关 系图. 显然光谱与温度之间具有很好的线性关系 $(R=$ 0.9999), 说明温度效应具有很好的规律性, 可以用于温 度的预测. 图 2(b)是葡萄糖浓度与 $z_{i}$ 之间的定量关系模 型, 可以发现样品浓度与 $z_{i}$ 之间存在较好的线性关系 $(R=0.9923)$, 利用此关系可进行葡萄糖浓度的定量预 测. 值得注意的是，上述计算结果是基于光谱在 8000 $6000 \mathrm{~cm}^{-1}$ 波数范围内水的光谱信息计算得到的, 说明 水结构的变化可以反映体系中溶质含量的变化, 同时也 表明温度的变化对水的结构具有影响, 并且近红外光谱 对水的结构变化非常敏感.
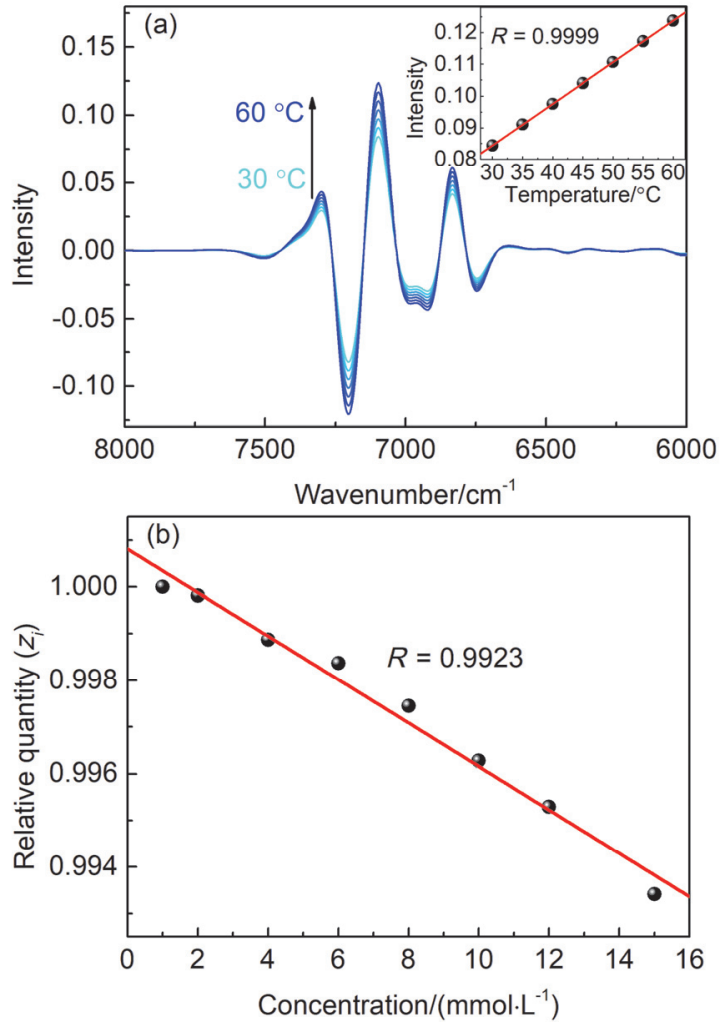

图 2 MFA 对 A 组样品光谱的计算结果: (a)标准信号(SS)和 7097 $\mathrm{cm}^{-1}$ 处的峰强度与温度之间的关系(嵌入图); (b)相对强度值 $\left(z_{i}\right)$ 与葡萄 糖浓度的关系

Figure 2 Results obtained by MFA for the spectra of the sample in group A. (a) Standardized signal (SS) and (inset) the relationship between the intensity at $7097 \mathrm{~cm}^{-1}$ and temperature. (b) Relationship between $z_{i}$ and glucose concentration
为了考察定量模型的预测能力, 配制了 $\mathrm{A}$ 组样品浓 度范围的 5 个样品进行了独立外部验证. 验证集样品配 制条件及近红外光谱测量条件均与校正集样品保持一 致. 对验证集样品光谱进行 CWT 预处理, 并计算每个 样品光谱的 $z_{i}$ 值, 通过图 2(b)的定量关系对葡萄糖的浓 度进行了预测. 图 3 是葡萄糖含量预测值与参考值之间 的关系, 其中黑色和红色点分别表示校正集样品和验证 集样品的预测结果. 通过分别对这些点进行线性拟合可 以发现, 两条线的斜率和截距很接近. 验证集样品预测 结果中葡萄糖浓度参考值和预测值的相关系数 $R$ 和 RMSEP 值分别为 0.9964 和 $0.35 \mathrm{mmol} / \mathrm{L}$. 此外, 将得到 的每个样品的误差及其对应的相对误差列于表 1 中. 观 察表中数据可以发现，参考值与预测值之间的最大误差 为 $0.62 \mathrm{mmol} / \mathrm{L}$. 结果表明, 模型具有很好的预测能力, 对于葡萄糖含量为 $1.0 \sim 15.0 \mathrm{mmol} / \mathrm{L}$ 的样品具有很好的 适用性.

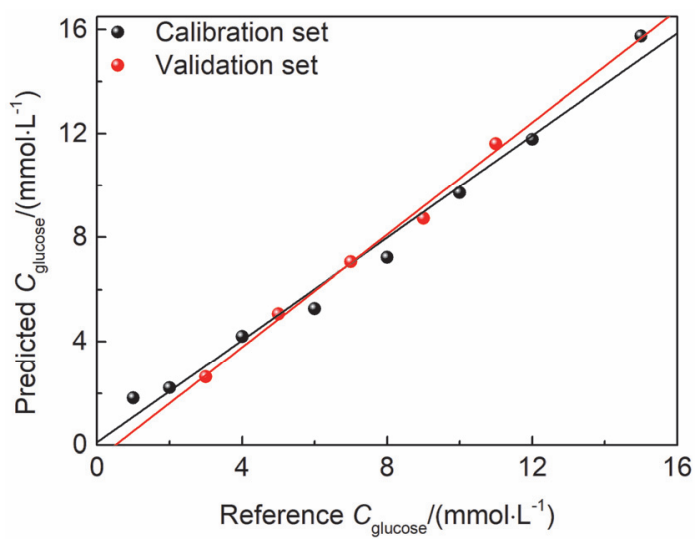

图 $3 \mathrm{~A}$ 组样品中葡萄糖含量参考值和预测值之间的关系(直线通过 线性拟合得到)

Figure 3 Relationship between the reference values and the predicted values of glucose content in group A samples (The line is obtained by linear fitting)

表 1 A 组验证集样品中葡萄糖含量的预测结果

Table 1 Prediction results of glucose content in the validation set samples of group A

\begin{tabular}{ccccc}
\hline 序号 & $\begin{array}{c}\text { 参考值/ } \\
\left(\mathrm{mmol} \cdot \mathrm{L}^{-1}\right)\end{array}$ & $\begin{array}{c}\text { 预测值/ } \\
\left(\mathrm{mmol} \cdot \mathrm{L}^{-1}\right)\end{array}$ & $\begin{array}{c}\text { 误差/ } \\
\left(\mathrm{mmol} \cdot \mathrm{L}^{-1}\right)\end{array}$ & 相对误差 $/ \%$ \\
\hline 1 & 3.0 & 2.64 & -0.36 & -12.00 \\
2 & 5.0 & 5.07 & 0.07 & 1.40 \\
3 & 7.0 & 7.06 & 0.06 & 0.86 \\
4 & 9.0 & 8.73 & -0.27 & -3.00 \\
5 & 11.0 & 11.62 & 0.62 & 5.64 \\
\hline
\end{tabular}

为了进一步验证 MFA 方法对更低浓度葡萄糖样品 的定量分析的适用性，对以血清为溶剂，葡萄糖浓度为 $0.0 \sim 1.0 \mathrm{mmol} / \mathrm{L}$ 的 $\mathrm{B}$ 组样品的光谱数据进行了相同的 计算, 结果如图 4 所示. 图 4(b)中葡萄糖浓度与 $z_{i}$ 值之 间的线性相关系数 $R$ 为 0.9895 , 表明 MFA 方法能够实 现对浓度为 $0.0 \sim 1.0 \mathrm{mmol} / \mathrm{L}$ 样品定量模型的建立.

为了进一步考察该模型的预测能力, 配制了 $\mathrm{B}$ 组样 品浓度范围的 6 个样品, 进行了独立外部验证. 对验证 

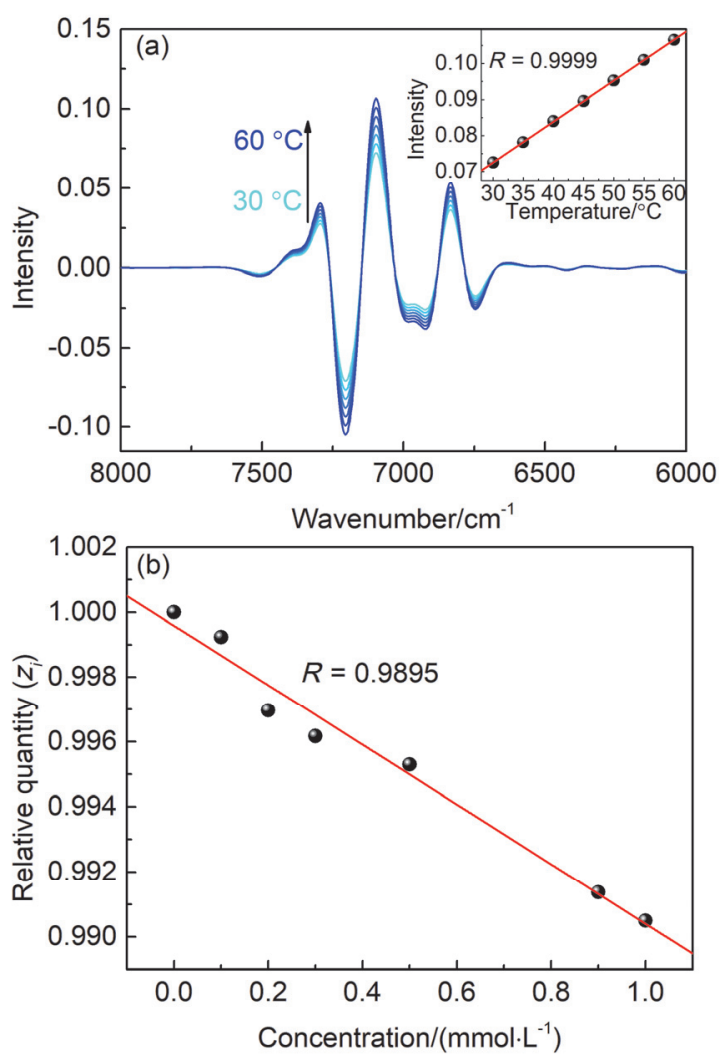

图 4 MFA 对 B 组样品光谱的计算结果: (a)标准信号(SS)和 7097 $\mathrm{cm}^{-1}$ 处的峰强度与温度之间的关系(嵌入图); (b)相对强度值 $\left(z_{i}\right)$ 与葡萄 糖浓度的关系

Figure 4 Results obtained by MFA for the spectra of the sample in group B. (a) Standardized signal (SS) and (inset) the relationship between the intensity at $7097 \mathrm{~cm}^{-1}$ and temperature. (b) Relationship between $z_{i}$ and glucose concentration

集样品实施同校正集样品相同的实验操作，包括样品配 制和光谱采集. 葡萄糖含量的预测值和参考值的关系如 图 5 所示, 其中黑色点表示校正集样品的预测结果, 红 色点表示验证集样品的预测结果. 验证集样品预测结果

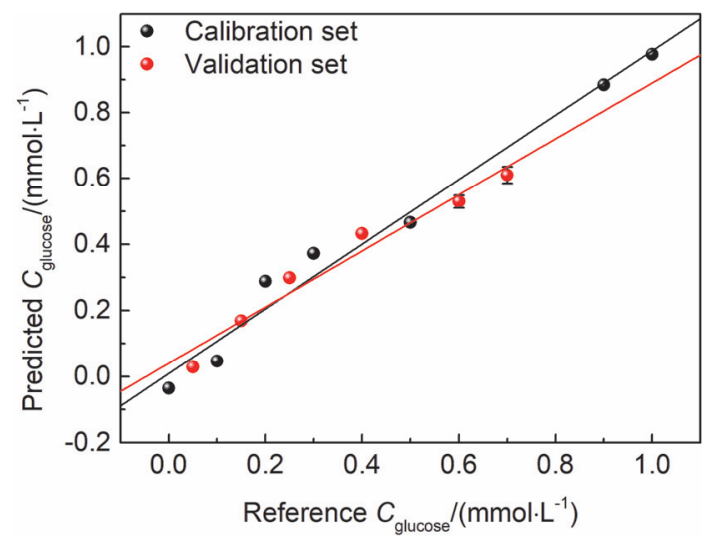

图 $5 \mathrm{~B}$ 组样品中葡萄糖含量参考值和预测值之间的关系(直线通过线 性拟合得到)

Figure 5 Relationship between the reference values and the predicted values of glucose content in group B samples (The line is obtained by linear fitting)
中葡萄糖含量参考值和预测值之间的相关系数 $R$ 和 RMSEP 值分别为 0.9833 和 $0.07 \mathrm{mmol} / \mathrm{L}$. 表 2 中数据为 验证集样品葡萄糖含量参考值与预测值之间的误差及 相对误差，可以发现最大误差为 $-0.09 \mathrm{mmol} / \mathrm{L}$ ，在临床 允许误差范围内 ${ }^{[31]}$. 但是 B 组验证集样品的相对误差均 较大, 葡萄糖浓度为 $0.05 \mathrm{mmol} / \mathrm{L}$ 样品的相对误差竟达 到 $40.00 \%$, 从相对误差方面来看, 建立的定量模型不适 用于较低浓度葡萄糖样品的预测. 此外, 为了考察方法 的重复性，利用验证集中葡萄糖浓度分别为 0.60 和 0.70 $\mathrm{mmol} / \mathrm{L}$ 的重复样品进行评估, 相对标准偏差(RSD)值分 别为 $3.77 \%$ 和 $4.92 \%$, 重复性良好. 以上结果表明, 模型 具有较好的预测能力，温控近红外光谱技术结合 MFA 方法可以实现血清中微量葡萄糖的定量检测.

表 2 B 组验证集样品中葡萄糖含量的预测结果

Table 2 Prediction results of glucose content in the validation set samples of group B

\begin{tabular}{ccccc}
\hline 序号 & $\begin{array}{c}\text { 参考值/ } \\
\left(\mathrm{mmol} \cdot \mathrm{L}^{-1}\right)\end{array}$ & $\begin{array}{c}\text { 预测值/ } \\
\left(\mathrm{mmol} \cdot \mathrm{L}^{-1}\right)\end{array}$ & $\begin{array}{c}\text { 误差/ } \\
\left(\mathrm{mmol} \cdot \mathrm{L}^{-1}\right)\end{array}$ & 相对误差 $/ \%$ \\
\hline 1 & 0.05 & 0.03 & -0.02 & -40.00 \\
2 & 0.15 & 0.17 & 0.02 & 13.33 \\
3 & 0.25 & 0.30 & 0.05 & 20.00 \\
4 & 0.40 & 0.43 & 0.03 & 7.50 \\
5 & 0.60 & 0.53 & -0.07 & -11.67 \\
6 & 0.70 & 0.61 & -0.09 & -12.86 \\
\hline
\end{tabular}

\section{3 结论}

通过温控近红外光谱技术研究了血清中微量葡萄 糖的定量分析. 采集了不同样品在不同温度下的近红外 光谱, 经过 CWT 改善光谱分辨率后, 应用 MFA 方法实 现了 $1.0 \sim 15.0 \mathrm{mmol} / \mathrm{L}$ 和 $0.0 \sim 1.0 \mathrm{mmol} / \mathrm{L}$ 两个浓度范 围葡萄糖样品的准确定量, 证明了该葡萄糖检测方法的 可行性和适用性. 因此，温控近红外光谱结合 MFA 方 法对于血清中微量葡萄糖的定量分析可能具有良好的 发展潜力.

\section{4 实验部分}

\section{1 试剂及样品制备}

葡萄糖(glucose，>99.5\%)购自上海阿拉丁生化科 技有限公司，人正常血清购自天津艾克泽生物科技有限 公司, 用于配制溶液. 配制了 $\mathrm{A}$ 和 B 两组样品, A 组样品 包含浓度范围在 $1.0 \sim 15.0 \mathrm{mmol} / \mathrm{L}$ 的 13 个样品, 其中 8 个样品来建立校正模型, 浓度分别为 $1.0,2.0,4.0,6.0$, $8.0,10.0,12.0$ 和 $15.0 \mathrm{mmol} / \mathrm{L}$, 其余 5 个样品则用来评价 模型的预测能力, 浓度分别为 3.0, 5.0, 7.0, 9.0 和 11.0 $\mathrm{mmol} / \mathrm{L} . \mathrm{B}$ 组样品包含浓度范围为 $0.0 \sim 1.0 \mathrm{mmol} / \mathrm{L}$ 的 13 个样品, 其中 7 个样品来建立校正模型, 浓度分别为 $0,0.1,0.2,0.3,0.5,0.9$ 和 $1.0 \mathrm{mmol} / \mathrm{L}$, 其余 6 个样品则用 来评价模型的预测能力, 浓度分别为 $0.05,0.15,0.25$, $0.4,0.6$ 和 $0.7 \mathrm{mmol} / \mathrm{L}$. 为了考察方法的重现性, 对于 B 
组验证集样品中 0.6 和 $0.7 \mathrm{mmol} / \mathrm{L}$ 的溶液分别配制了 3 个重复样品. 在光谱测量前将血清置于 $-20{ }^{\circ} \mathrm{C}$ 冷冻保 存, 以保持其化学成分的完整性.

\section{2 温度控制和光谱采集}

采用 Vertex 70 光谱仪(Bruker Optics Inc, Ettlingen, Germany)测量近红外透射光谱. 采集近红外光谱时使用 光程为 $1 \mathrm{~mm}$ 的石英比色皿. 天津艾利安电子科技有限 公司)、卤素铇灯光源和 $\mathrm{InGaAs}$ 检测器测, 所采光谱范 围为 $12000 \sim 4000 \mathrm{~cm}^{-1}$, 光谱分辨率为 $8 \mathrm{~cm}^{-1}$, 采样间 隔约为 $4 \mathrm{~cm}^{-1}$, 扫描次数为 64 次.

实验中的温度由 $2216 \mathrm{e}$ 型温度控制器(Bruker Optics Inc, Ettlingen, Germany)控制, 精度为 $\pm 0.1{ }^{\circ} \mathrm{C}$. 以空比 色具为背景, 在 $30 \sim 60{ }^{\circ} \mathrm{C}$ 温度范围内间隔为 $5{ }^{\circ} \mathrm{C}$, 采 集 $\mathrm{A}$ 和 $\mathrm{B}$ 两组样品的近红外光谱, 测量时将比色㿼密 封, 每个温度下稳定 $20 \mathrm{~min}$ 后进行光谱采集.

\section{3 计算}

CWT 通常用来消除背景漂移和增强光谱分辨

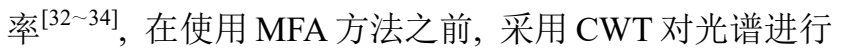
预处理. 利用消失矩为 6 的 Symmlet 小波基(Sym6)进行 连续小波变换, 近似于对光谱求六阶导数 ${ }^{[7,27]}$, 尺度系 数为 25 .

MFA 是一种提取和比较不同样品光谱数据中共同 包含的光谱信息的化学计量学方法, 可实现样品中物质 含量的定量分析 ${ }^{[7]}$. 进行 MFA 计算时, 首先将不同样品 在不同温度下测得的光谱按照浓度横向排列构成组合 数据矩阵, 然后对组合数据矩阵进行 PCA (Principal Component Analysis)计算, 提取组合数据矩阵中共同包 含的光谱信息, 以每个样品组中葡萄糖浓度最低的样品 作为参考, 得到标准信号 $(\mathrm{SS}), \mathrm{SS}$ 体现了光谱随温度的 变化, 而 SS 在不同浓度样品光谱中的相对强度(即 $z_{i}$ 值) 体现了浓度的相对高低. 最后建立浓度与 $z_{i}$ 之间的定量 模型，从而达到定量分析的目的.

\section{References}

[1] Sharma, S.; Goodarzi, M.; Delanghe, J.; Ramon, H.; Saeys, W. Appl.
Spectrosc. 2014, 68, 398.

[2] Chu, M. X.; Miyajima, K.; Takahashi, D.; Arakawa, T.; Sano, K.; Sawada, S.; Kudo, H.; Iwasaki, Y.; Akiyoshi, K.; Mochizuki, M.; Mitsubayashi, K. Talanta 2011, 83, 960.

[3] Hazen, K. H.; Arnold, M. A.; Small, G. W. Appl. Spectrosc. 1994, $48,477$.

[4] Chen, J.; Arnold, M. A.; Small, G. W. Anal. Chem. 2004, 76, 5405.

[5] Liu, L. Z.; Arnold, M. A. Anal. Bioanal. Chem. 2009, 393, 669.

[6] Cui, X. Y.; Liu, X. W.; Yu, X. M.; Cai, W. S.; Shao, X. G. Anal. Chim. Acta 2017, 957, 47.

[7] Shao, X. G.; Cui, X. Y.; Yu, X. M.; Cai, W. S. Talanta 2018, 183, 142.

[8] Marquardt, L. A.; Arnold, M. A.; Small, G. W. Anal. Chem. 1993, 65, 3271 .

[9] Sharma, S.; Goodarzi, M.; Delanghe, J.; Ramon, H.; Saeys, W. Appl. Spectrosc. 2014, 68, 398.

[10] Goodarzi, M.; Saeys, W. Talanta 2016, 146, 155.

[11] Hazen, K. H.; Arnold, M. A.; Small, G. W. Anal. Chim. Acta 1998, $371,255$.

[12] Amerov, A. K.; Chen, J.; Small, G. W.; Arnold, M. A. Anal. Chem. 2005, 77, 4587.

[13] Kang, N.; Kasemsumran, S.; Woo, Y.; Kim, H.; Ozaki, Y. Chemom Intell. Lab. Syst. 2006, 82,90

[14] Tsenkova, R. NIR News 2008, 19, 12.

[15] Tsenkova, R. J. Near Infrared Spectrosc. 2009, 17, 303.

[16] Tarumi, M.; Shimada, M.; Murakami, T.; Tamura, M.; Shimada, M.; Arimoto, H.; Yamada, Y. Phys. Med. Biol. 2003, 48, 2373.

[17] Cui, X. Y.; Cai, W. S.; Shao, X. G. RSC Adv. 2016, 6, 105729.

[18] Cheng, D.; Cai, W. S.; Shao, X. G. Appl. Spectrosc. 2018, 72, 1354.

[19] Wang, L.; Zhu, X. W.; Cai, W. S.; Shao, X. G. Phys. Chem. Chem. Phys. 2019, 21, 5780 .

[20] Zhu, X. W.; Cui, X. Y.; Cai, W. S.; Shao, X. G. Acta Chim. Sinica 2018, 76, 298. (in Chinese). (朱雪薇, 崔晓宇, 蔡文生, 邵学广, 化学学报, 2018, 76, 298.)

[21] Fan, M. L.; Cai, W. S.; Shao, X. G. Appl. Spectrosc. 2016, 71, 472.

[22] Liu, X. W.; Cui, X. Y.; Yu, X. M.; Cai, W. S.; Shao, X. G. Chin. Chem. Lett. 2017, 28, 1447.

[23] Ma, L.; Cui, X. Y.; Cai, W. S.; Shao, X. G. Phys. Chem. Chem. Phys. 2018, 20, 20132.

[24] Qi, L. H.; Cai, W. S.; Shao, X. G. Acta Chim. Sinica 2016, 74, 172. (in Chinese). (祁丽华, 蔡文生, 郡学广, 化学学报, 2016, 74, 172.)

[25] Shao, X. G.; Kang, J.; Cai, W. S. Talanta 2010, 82, 1017.

[26] Kang, J.; Cai, W. S.; Shao, X. G. Talanta 2011, 85, 420.

[27] Shao, X. G.; Cui, X. Y.; Wang, M.; Cai, W. S. Spectrochim. Acta Part $A$ 2019, 213, 83.

[28] Czarnecki, M. A.; Morisawa, Y.; Futami, Y.; Ozaki, Y. Chem. Rev. 2015, 115, 9707.

[29] Šašić, S.; Segtnan, V. H.; Ozaki, Y. J. Phys. Chem. A 2002, 106, 760.

[30] Segtnan, V. H.; Šašić, S.; Isaksson, T.; Ozaki, Y. Anal. Chem. 2001, $73,3153$.

[31] Rajendran, R.; Rayman, G. J. Diabetes Sci. Technol. 2014, 8, 1081

[32] Shao, X. G.; Cai, W. S. Rev. Anal. Chem. 1998, 17, 235.

[33] Shao, X. G.; Leung, A. K. M.; Chau, F. T. Acc. Chem. Res. 2003, 36, 276.

[34] Shao, X. G.; Ma, C. X. Chemom. Intell. Lab. Syst. 2003, 69, 157.

(Cheng, B.) 